\title{
Value of High-frequency Ultrasonography with Virtual Touch Tissue Quantification in Diagnosis of Breast Pure Mucinous Carcinomas
}

\author{
Shi Jian*, Yang Guang and Tao Ye \\ Department of Ultrasound, The First Affiliated Hospital of Dalian Medical University, Dalian, China
}

\begin{abstract}
Objective: To analyze the characters of breast pure mucinous carcinomas on high-frequency ultrasonography with Virtual Touch Tissue Quantification (VTQ).

Methods: A total of 12 patients (with breast pure mucinous carcinomas) and a group of 30 patients (with adenofibroma of breast) underwent breast examination with high-frequency ultrasonography to analyze the characters of images, and with VTQ to analyze the elastic character.

Results: In the conventional ultrasound imaging, statistical differences were found between two groups in the shape, the boundary and the internal echo of the lesions. In the VTQ, the mean of shearing wave speed (Vs) in pure mucinous carcinomas is less than in adenofibroma of breast.
\end{abstract}

Conclusion: Conventional high-frequency ultrasonography combining with VTQ have significant value in diagnosis of breast pure mucinous carcinoma.

Keywords: Breast; Pure mucinous carcinoma; Ultrasonography

\section{Introduction}

Breast mucinous carcinoma is a malignant breast tumor in a rare pathological type, accounting for all breast cancer 1 to 6\% [1], due to special pathological changes and similarity in conventional ultrasound images and clinical manifestation to benign fibroadenoma which would be misdiagnosed. Virtual touch tissue quantification (VTQ) imaging, a newly developed elastography, instead of using external compression, evaluates tissue quantitatively to providing complementary informations to conventional ultrasound. This study used the conventional high-frequency ultrasound combined with VTQ technology on the breast of pure mucinous adenocarcinoma comparison with fibroadenoma, and inspected, observated and compared the sonographic features and flexibility characteristics in order to improve understanding of pure mucinous adenocarcinoma of the breast [2].

\section{Information and Methods}

\section{The object of study}

Ultrasound was performed in the patients who underwent evaluation of breast lesions at our department from November 2010 to December 2011 and confirmed by pathology, 12 cases of breast patients with pure mucinous adenocarcinoma and 30 cases of breast patients with fibroadenoma were analyzed retrospectively, patients were women, pure mucinous adenocarcinoma group aged 42 to 83 years old, average $61.1 \pm 12.8$ years; a fibroadenoma age group 33 to 56 years, an average of $38.2 \pm 10.9$ years.

\section{Instruments and methods}

Application of Siemens ACUSON S2000 ultrasound diagnostic apparatus with Acoustic Radiation Force, Impulse (ARFI) technology, high-frequency linear array probe 9L4, frequency $4 \sim 9 \mathrm{MHz}$.

Conventional ultrasound scanning was performed on the patients in the supine position, fully exposed breast, the nipple as the center, radial vertical and horizontal cut scanning in all quadrants, and according to the specific circumstances of the lesion adjusted the depth, gain, focus location of the images. The ultrasound characteristics including lesion shape, edge, border, traveling direction, internal echo and posterior echo were obtained.

VTQ was performed in the VTQ mode of ARFI technology by the same machine and the same transducer which was applied with light pressure to make complete contact with the breast but to let the patient in breath-hold. The region-of-interest (ROI) (size $0.5 \mathrm{~cm} \times 0.5 \mathrm{~cm}$ ) was placed entirely in the lesion, and the maximum depth of $5.5 \mathrm{~cm}$. The shear wave velocity (Vs) was measured in the lesion, the simultaneous measures in peritumoral glandular tissue (from the lesion $1 \sim 2 \mathrm{~cm}$ ) and the normal glandular tissue of the same depth were performed, every location was measured 10 times, take the results to calculate the average. All images and data are stored in the CD analysis.

\section{Statistical analysis}

Application of SPSS 13.0 statistical software for data analysis, in the conventional ultrasound imaging the differences of characteristics between pure breast mucinous adenocarcinoma group and fibroadenoma group were analyzed using the $\chi^{2}$ test; in the VTQ technology, the VTQ value (Vs) using the mean \pm standard deviation, the difference between the two groups was analyzed by group $t$ test, relationships between VTQ variables of different location were examined using $q$ test, $P<0.05$ was considered statistically significant difference.

*Corresponding author: Shi Jian, Department of Ultrasound, The First Affiliated Hospital of Dalian Medical University, Dalian, China, E-mail: smqd76@hotmail.com

Received February 03, 2012; Accepted February 15, 2012; Published February 17,2012

Citation: Jian S, Guang Y, Ye T (2012) Value of High-frequency Ultrasonography with Virtual Touch Tissue Quantification in Diagnosis of Breast Pure Mucinous Carcinomas. J Cancer Sci Ther 4: 043-045. doi:10.4172/1948-5956.1000108

Copyright: (c) 2012 Jian S, et al. This is an open-access article distributed under the terms of the Creative Commons Attribution License, which permits unrestricted use, distribution, and reproduction in any medium, provided the original author and source are credited. 
Citation: Jian S, Guang Y, Ye T (2012) Value of High-frequency Ultrasonography with Virtual Touch Tissue Quantification in Diagnosis of Breast Pure Mucinous Carcinomas. J Cancer Sci Ther 4: 043-045. doi:10.4172/1948-5956.1000108

\section{Results}

\section{A conventional high-frequency ultrasound performance}

Comparison of conventional ultrasound performance in 12 cases of breast pure mucinous adenocarcinoma and 30 cases of fibroadenoma are shown in Table 1, differences in the shape, the boundary and internal echo were statistically significant $(\mathrm{P}<0.05)$ (Figure 1,2$)$.

\section{VTQ}

The comparison of VTQ values (Vs) in different location of two groups are shown in the Table 2. The difference of Vs in the lesion between the two groups was significant $(\mathrm{t}=8.42, \mathrm{P}<0.01)$ (Figure

\begin{tabular}{|c|c|c|c|c|c|}
\hline \multicolumn{2}{|c|}{ Ultrasonography } & $\begin{array}{l}\text { Pure mucinous } \\
\text { adenocarcinoma }\end{array}$ & Fibroadenoma & $\begin{array}{c}x^{2} \\
\text { value }\end{array}$ & $P$ value \\
\hline \multirow{2}{*}{ Shape } & Round or oval & 3 & 24 & \multirow{2}{*}{8.60} & \multirow{2}{*}{$<0.005$} \\
\hline & Irregular in shape & 9 & 6 & & \\
\hline \multirow{2}{*}{ Edge } & Clear & 8 & 27 & \multirow{2}{*}{3.36} & \multirow{2}{*}{0.072} \\
\hline & Blurred or burr-like & 4 & 3 & & \\
\hline \multirow{2}{*}{ Boundary } & Interface sharp & 5 & 29 & \multirow{2}{*}{16.82} & \multirow{2}{*}{$<0.005$} \\
\hline & Strong echo halo & 7 & 1 & & \\
\hline \multirow{2}{*}{$\begin{array}{c}\text { Mass } \\
\text { orientation }\end{array}$} & Parallel & 7 & 25 & \multirow{2}{*}{2.86} & \multirow{2}{*}{0.12} \\
\hline & Not parallel & 5 & 5 & & \\
\hline \multirow{2}{*}{$\begin{array}{l}\text { Internal } \\
\text { echo }\end{array}$} & Uniform & 3 & 21 & \multirow{2}{*}{7.08} & \multirow{2}{*}{$<0.01$} \\
\hline & Uneven & 9 & 9 & & \\
\hline \multirow{2}{*}{$\begin{array}{l}\text { Posterior } \\
\text { echo }\end{array}$} & Increase & 7 & 22 & \multirow{2}{*}{0.90} & \multirow{2}{*}{0.34} \\
\hline & Does not enhance & 5 & 8 & & \\
\hline
\end{tabular}

Table 1: Comparison of conventional ultrasound performance table of breast pure mucinous adenocarcinoma and breast fibroadenoma.

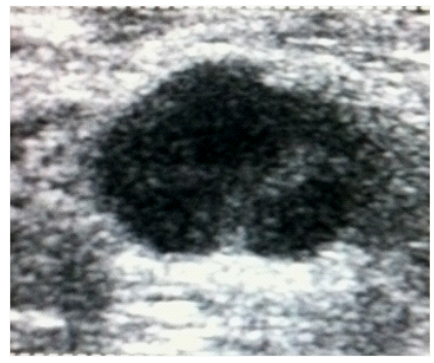

Figure 1: Conventional high-frequency ultrasound image of a 50-year-old woman named Yu Guilan with a pure mucinous carcinoma of breast: irregular shape, clear margins, no capsule, showing the echogenic halo sign, hypoechoic internal echo unevenly, posterior echo enhancement.

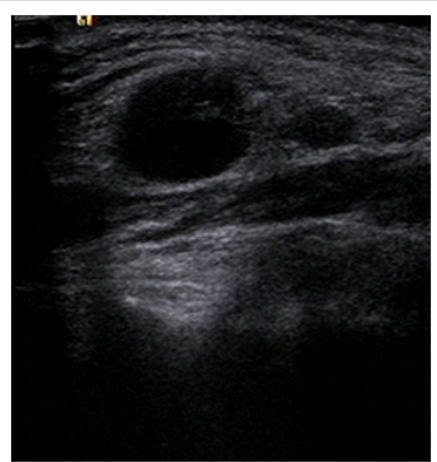

Figure 2: Conventional high-frequency ultrasound image of a 39-year-old woman named Li Yan with breast fibroadenoma: regular shape, clear margins, complete envelope, low internal echo uniformly, posterior echo enhancement.

\begin{tabular}{|c|c|c|c|c|c|c|}
\hline \multirow{2}{*}{ Breast tumor } & \multirow{2}{*}{$\begin{array}{l}\text { The } \\
\text { number } \\
\text { of cases }\end{array}$} & \multicolumn{3}{|c|}{ Different location Vs $(\mathrm{m} / \mathrm{s}, \bar{x} \pm s)$} & \multicolumn{2}{|l|}{$P$ values } \\
\hline & & $\begin{array}{l}\text { Inside the } \\
\text { lesion }\end{array}$ & peritumoral & Normal & \begin{tabular}{|c|} 
Internal / \\
peritumoral
\end{tabular} & $\begin{array}{c}\text { peritumoral/ } \\
\text { normal }\end{array}$ \\
\hline $\begin{array}{l}\text { Pure mucinous } \\
\text { adenocarcinoma }\end{array}$ & 12 & $1.15 \pm 0.75$ & $2.03 \pm 0.43$ & $2.32 \pm 0.46$ & $<0.001$ & 0.078 \\
\hline Fibroadenoma & 30 & $3.27 \pm 0.56$ & $2.37 \pm 0.66$ & $2.24 \pm 0.37$ & $<0.001$ & 0.14 \\
\hline
\end{tabular}

Table 2: VTQ values of two groups and each group of lesions in different location.

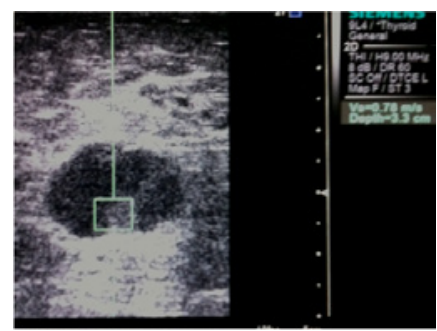

Figure 3: Ultrasound ARFI technique with VTQ value in a breast pure mucinous carcinoma of a 50 -year-old woman named Yu Guilan: Vs $=0.78 \mathrm{~m} / \mathrm{s}$

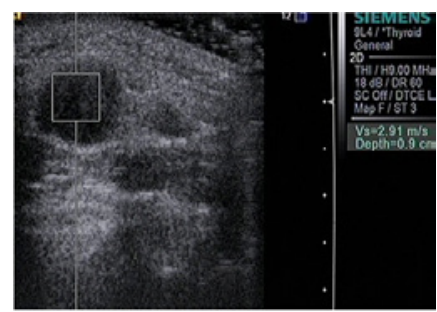

Figure 4: Ultrasound ARFI technique with VTQ value in a breast fibroadenoma of a 39-year-old woman named Li Yan: Vs $=2.91 \mathrm{~m} / \mathrm{s}$.

3,4). In each group, the difference of Vs between inside the lesion and peritumoral glandular tissue was significant. While the difference of Vs between peritumoral glandular tissue and normal glandular tissue was not significant.

\section{Discussion}

Breast mucinous carcinoma, also known as colloid breast cancer, ductal epithelium, is a rare type of invasive breast cancer occurred in postmenopausal women over the age of 50 and clinical palpation texture of soft or tough, active, adhesion flu. The characteristics of mucinous carcinoma are extracellular mucus in the tumor, and the mucous component in pure mucinous carcinoma accounts for $75 \%$ or more. The tumor with smooth surface, clear boundary and expansive growth slowly has lower metastasis and significantly better prognosis than the other common types of breast cancers [3].

On the performance of conventional high-frequency ultrasound on the breast pure mucinous adenocarcinoma is low echo, clear edge and more enhanced effect in posterior echo, similar to the characteristics of these in fibroadenoma. In this study, mostly irregular breast pure mucinous adenocarcinoma, was Oita leafy, clear most of the tumor edge, no envelope boundary expressed as echogenic halo sign, and fibroadenoma of breast are mostly round or oval shape, with intact membrane, smooth, sharp boundary [4]. The internal echo of most of fibroadenoma was uniform low echo, while internal echo of pure mucinous adenocarcinoma is low or very low, mostly heterogeneous echo, which have smaller scattered distribution of echo-free zone, which was considered led to riching in mucus, and in mucinous adenocarcinoma related study [5], the mass no apparent liquid zone, 
but showed cord-like hyperechoic and hypoechoic, disorganized nonhomogeneous region, is one of the characteristics of ultrasound images of breast mucinous carcinoma.

ARFI, a recently developed ultrasound elastography technique, uses probes to generate short-duration, high-intensity, acoustic pulse to generate localized, micron-scale displacements of tissue within the ROI, VTQ tracks a shear wave within the ROI that travels perpendicular to the transmitted longitudinal push pulse. Time to Peak analysis computes a numerical value of the shear wave speed obtained over the ROI which objectively reflects the hardness or elasticity of the target tissue, the greater the value, the stiffer a tissue. Krouskop et al. [6] showed that various breast tissues had different elastic stiffness, invasive carcinoma having the lowest elasticity, followed by noninvasive carcinoma, fibrous tissue in the breast, normal glandular breast tissue, and breast fat tissue in that order. Meng et al. [7] reported in 2011, VTQ values were significantly higher in malignant than benign breast lesions, and Vs are higher in both malignant and benign breast lesions than normal breast tissue, but most of the malignant tumors were invasive ductal carcinoma, not including a mucinous carcinoma. Elastography of breast tissue hardness is divided into five grades from low to high previously [8], the hardness of mucinous carcinoma is less than 3 grade. In this study, VTQ value of pure mucinous carcinoma of breast was lower than the surrounding normal breast tissue, was also significantly lower than the fibroadenoma. Fibroadenoma occurred in the end of the lobular duct and formed due to proliferation of interstitial cells and endothelial cells, stromal hyperplasia is more obvious. While the main component of pure mucinous adenocarcinoma is mucus, the based histopathological differences may be the hardness of fibroadenoma higher than pure mucinous adenocarcinoma. In this study, the difference of Vs of pure mucinous adenocarcinoma between normal glandular tissue and the peripheral tissue was not significant, which is different from the ordinary invasive ductal carcinoma from Meng et al. [7], who pointed out that Vs value of the peripheral tissues of invasive ductal carcinoma is significantly higher than the normal glandular tissue, reflecting the tissue of cancer infiltration to the surrounding glands. Pure mucinous adenocarcinoma was expansive growth and less infiltration, so it may be relevant to its prognosis of lower transfer rate.

As ARFI imaging generates radiation pulse from the probe to promote the local organization and result to a small deformation, compared to the other elasticity imaging techniques, ARFI takes advantages to not rely on the operator to push the organization, not be affected by adjacent hard tissue and be higher repetitive. VTQ technology can quantify the extent of flexibility of the organization and thus more objectively reflect the organization's flexibility or hardness. Because incidence of breast pure mucinous adenocarcinoma is low and small sample size of this study, the Vs value would be biased, yet it needed to increase the sample size in order to further study of ARFI technology to the value of clinical diagnosis of breast mucinous carcinoma.

Simple summary, in conventional high-frequency ultrasound, breast pure mucinous carcinoma has the shape more irregular, boundary hyperechoic halo sign and heterogeneous inner echo texture which can be used as the identification of points with the fibroadenoma. On the basis of conventional ultrasound, combination of the VTQ technology objectively reflects the elastic characteristics of tissue. Our result, the hardness of pure mucinous adenocarcinoma is lower than fibroadenoma, provides complementary information for the differential diagnosis of mucinous carcinoma, but also to make up for the awareness to breast elastic characteristics of the type of rare malignant tumor.

\section{References}

1. Komenaka IK, El-Tamer MB, Troxel A, Hamele-Bena D, Joseph KA, et al. (2004) Pure mucinous carcinoma of the breast. Am J Surg 187: 528-532.

2. Yulan $P$ (2004) High-frequency breast ultrasound map. (1st edn), People's Health Publishing House, Beijing.

3. Qunjun F, Jun J, Fei Z (2003) Clinical and pathological features of breas mucinous carcinoma. Third Military Medical University 25: 2077-2079.

4. Capella C, Eusebi V, Mann B, Azzopardi JG (1980) Endocrine differentiation in mucoid carcinoma of the breast. Histopathology 4: 613-630.

5. Lam WW, Chu WC, Tse GM, Ma TK (2004) Sonographic appearance of mucinous carcinoma of the breast. AJR Am J Roentgenol 182: 1069-1074.

6. Krouskop TA, Wheeler TM, Kallel F, Garra BS, Hall T (1998) Elastic moduli of breast and prostate tissues under compression. Ultrason Imaging 20: 260-274.

7. Meng W, Zhang G, Wu C, Wu G, Song Y, et al. (2011) Preliminary results of acoustic radiation force impulse (ARFI) ultrasound imaging of breast lesions. Ultrasound Med Biol 37: 1436-1443.

8. Itoh A, Ueno E, Tohno E, Kamma H, Takahashi H, et al. (2006) Breast disease: clinical application of US elastography for diagnosis. Radiology 239: 341-350. 